\title{
Incremento de la temperatura ambiental y su posible asociación al suicidio en Baja California Sur (BCS) 1985-2008
}

\author{
Ramón Gaxiola-Robles, ${ }^{1}$ Alfredo de Jesús Celis de la Rosa, ${ }^{2}$ Vanessa Labrada-Martagón, ${ }^{3}$ \\ Sara Cecilia Díaz-Castro, ${ }^{3}$ Tania Zenteno-Savín ${ }^{3}$
}

Artículo original

\section{SUMMARY}

Introduction

During the last decade we have observed important climate changes, especially in environmental temperatures. There is considerable information linking the increase in hot weather and human health. For example, hot weather is associated with an increased risk of suicide in different countries around the world.

\section{Objective}

To evaluate the relationship between suicide rates and the environmental temperature in Baja California Sur, Mexico, from 1985 to 2008.

\section{Method}

Suicide mortality data for Baja California Sur (BCS) were obtained for the years 1985-2008 from the Instituto Nacional de Estadística, Geografía e Informática (INEGI). The selected codes were: E950-E959 (ICD-9) and X60-X84, Y87.0 (ICD-10) for BCS. The BCS weather data used was the maximum temperature from 1985 to 2008 , obtained from the Extractor Rápido de Información Climatológica (ERIC III). Lineal and quadratic models were used to assess the annual rate changes of suicide and generalized lineal models (GLM) to assess the effect of the climatological variables to the suicide rate. The $p \leq 0.05$ was considered significant.

\section{Results}

In BCS, 582 suicide deaths were reported from 1985 to 2008 . The $9 \%(53)$ of the total reported were women with a yearly average rate $1.6 / 100000 ; 91 \%(529)$ were men with a yearly average rate $16.3 / 100000$. Lineal and quadratic models explained the tendency of the annual increment observed in the number of suicides in both seasons. The quadratic model better explained such increment during the warmer months $\left(R^{2}=0.64 p<0.01\right)$. The temperature was positively correlated with the rate of suicides in both seasons $(p<0.01)$. Two predictive GLMs were created by season.

\section{Discussion}

These results suggest a potential link between an increase in environmental temperature and the rates of suicide during 24 years in BCS. This relationship is clear during the hot season; however, a positive trend was found during the cold season, perhaps due to the result of warmer winters.

Key words: Climate changes, suicide, Baja California Sur.

\section{RESUMEN}

Introducción

Durante las últimas décadas se ha podido apreciar un cambio en las variables climáticas, en especial en la temperatura ambiental. Hay evidencias que asocian el aumento de la temperatura ambiental con el incremento en las tasas de mortalidad por suicidio.

\section{Objetivo}

Evaluar la relación entre la tasa de suicidio y la temperatura ambiental en Baja California Sur (BCS) durante los años 1985-2008.

\section{Método}

Los datos de suicidio fueron obtenidos de las bases de Mortalidad del INEGI de 1985-2008. Se tabularon los registros con códigos: E950-E959 CIE-9 y X60-X84, Y87.0 CIE-10 para BCS. En el análisis climático se usaron las temperaturas máximas de 1985 al 2008 para BCS del Extractor Rápido de Información Climatológica (ERIC III). Para evaluar la tendencia, se utilizaron modelos de regresión lineal y cuadráticos. Los modelos lineales generalizados (GLM) se utilizaron para evaluar el efecto de las variables ambientales sobre la tasa de suicidios, con una significancia de $p \leq 0.05$.

\section{Resultados}

Un total de 582 suicidios ocurrieron en BCS de 1985 a 2008. Las mujeres representaron el $9 \%(53)$, con tasa anual promedio de $1.6 / 100000$. Los hombres $91 \%$ (529), con tasa promedio anual de 16.3/100000. La tendencia de incremento anual se explicó con modelos lineales simples y cuadráticos en ambas temporadas. En los meses cálidos el modelo cuadrático explicó mejor dicho incremento $\left(R^{2}=0.64 p<0.01\right)$. La temperatura se correlacionó positivamente con la tasa en ambas temporadas $(p<0.01)$. Se generaron dos modelos GLM predictivos por temporada.

\section{Discusión}

En este trabajo se observó una posible relación entre el incremento de la temperatura ambiental y el número de suicidios registrados durante 24 años en BCS. Esta relación se encuentra bien definida en los meses cálidos, pero con una tendencia en aumento para los meses fríos.

Palabras clave: Cambio climático, suicidio, Baja California Sur.

\footnotetext{
Centro de Investigaciones Biológicas del Noroeste (CIBNOR), Planeación Ambiental y Conservación, La Paz, Baja California Sur, México. Hospital General de Zona No.1, Instituto Mexicano del Seguro Social, La Paz, Baja California Sur, México.

2 Departamento de Salud Pública, Universidad de Guadalajara y Unidad de Investigación Médica en Epidemiologia Clínica, Hospital de Especialidades del IMSS Guadalajara, Jalisco, México.

3 Centro de Investigaciones Biológicas del Noroeste (CIBNOR), Planeación Ambiental y Conservación, La Paz, Baja California Sur, México.

Correspondencia: Tania Zenteno-Savín CIBNOR. Planeación Ambiental y Conservación. Instituto Politécnico Nacional 195, Playa Palo de Santa Rita Sur, 23096 La Paz, B.C.S. México. Teléfono:(612) 123 - 8484, Ext. 3341 y 3718, (612) 123 - 8502. Fax: (612) 125 - 3625. E-mail: tzenteno04@cibnor.mx

Recibido primera versión: 12 de junio de 2012. Segunda versión: 18 de abril de 2013. Aceptado: 31 de julio de 2013
} 


\section{INTRODUCCIÓN}

La idea de relacionar los aspectos climatológicos con la salud humana es tan antigua como la humanidad misma. Hace aproximadamente 2400 años, Hipócrates se refirió a las alteraciones meteorológicas y la salud. Escribió en su obra De los aires, las aguas y los lugares: "quienquiera que desee investigar adecuadamente en medicina, debe proceder así: en primer lugar debe de tener en cuenta las estaciones del año y los efectos que cada una produce; luego, los vientos, el calor y el frío...". ${ }^{1}$

A la fecha, múltiples trabajos han tratado de relacionar la salud humana con los factores climáticos, en especial cómo afectan éstos a la morbilidad y a la mortalidad. ${ }^{2,3}$ La evidencia epidemiológica demuestra que el incremento de la temperatura ambiental causa estrés térmico, incremento en las enfermedades infecciosas y destrucción de los cultivos, muerte del ganado y por consiguiente hambrunas. ${ }^{4}$

El calor afecta a los individuos con respuestas adaptativas limitadas; entre estos grupos vulnerables se encuentran los ancianos e individuos con enfermedades crónicas. ${ }^{5}$ En Italia se realizó un estudio de 1974 a 2003 en el cual se asocia el calentamiento global con el incremento de riesgo de suicidio. ${ }^{5}$ Asimismo, se ha relacionado la presencia de ondas de calor al incremento en el número de suicidios en la población del Reino Unido. ${ }^{6}$ Pero en ambos casos se trata de países con climas templados, por lo que queda la pregunta de ¿Qué pasa en lugares tropicales o realmente calientes? ${ }^{7}$

El suicidio en Baja California Sur (BCS) ha sido abordado desde el contexto nacional, en el cual lo sitúan dentro de los cinco primeros lugares. ${ }^{8}$ A pesar de la magnitud del evento para la entidad, éste sería apenas el segundo trabajo que aborda el problema desde el punto de vista epidemiológico. En el trabajo previo, se reportó que los hombres son los que más se suicidan en comparación con las mujeres. El matrimonio en los grupos de edad menores de 43 años confiere un efecto de reducción del riesgo. Posterior a los 44 años, los individuos casados son los más vulnerables. En cuanto al tamaño de la población, comunidades menores de 20000 habitantes son las que presentan el mayor riesgo. ${ }^{9}$

BCS es una entidad federativa de la República Mexicana donde el clima es caliente y árido. ${ }^{10}$ La región está influenciada por factores meteorológicos diversos y se ha podido evidenciar el calentamiento global en las últimas décadas. ${ }^{11}$ El incremento de la temperatura ambiental ha sido de $0.74^{\circ} \mathrm{C}$ y asciende rápidamente. En un estado donde la limitante del recurso hídrico ha sido determinante para su desarrollo, el incremento en intensidad y duración de las sequías está provocando muerte del ganado y fauna silvestre, así como la disminución del abastecimiento de agua y disfunción de las áreas de cultivo. ${ }^{11}$

Por lo anterior, se estableció como objetivo de este trabajo evaluar la relación entre la tasa de suicidio y la temperatura ambiental en BCS durante los años 1985-2008.

\section{MÉTODO}

Para la realización de este trabajo, se planteó un estudio de corte ecológico. En este tipo de estudios se trabaja con el nivel de exposición de varios grupos de individuos (o del mismo grupo en diferentes periodos de tiempo); la información es agregada en bases de datos a nivel de unidad geográfica. ${ }^{12}$ Para el presente estudio, se utilizaron datos de suicidio obtenidos de las bases de mortalidad del Instituto Nacional de Estadística, Geografía e Informática (INEGI) de los años 1985 a $2008 .{ }^{13}$ De los registros de mortalidad a nivel nacional, sólo se tabularon los correspondientes a BCS y de edad mayor a 14 años, según los códigos E950-E959 de la $9^{\circ}$ revisión de la Clasificación Internacional de Enfermedades (CIE-9 $)^{14}$ y X60-X84, Y87.0 de la $10^{\circ}$ revisión (CIE-10). ${ }^{15} \mathrm{La}$ selección de sujetos mayores de 14 años se debe a que las tasas de suicidio se tornan consistentes en la adolescencia tardía en los hombres y a la mitad de ésta en las mujeres. ${ }^{16,17}$ Las variables consideradas en los casos de defunción fueron la edad, sexo y el lugar del evento.

Para la creación de la variable climática, se usaron los datos diarios de temperatura máxima de 1985 a 2008 para BCS. Los datos fueron extraídos de la base denominada Extractor Rápido de Información Climatológica (ERIC III), creada por el Instituto Mexicano de Tecnología del Agua. Dichos registros se actualizaron con los datos del Servicio Meteorológico Nacional. ${ }^{18} \mathrm{El}$ estudio se delimitó a 24 años debido a que la información de las bases de datos sobre suicidio en fechas anteriores a 1985 sólo contaba con el año del evento, más no del mes; esto limitó el establecimiento de la temporalidad del evento. Las tasas de mortalidad por suicidio se calcularon como el número total de muertes por dicha causa ocurridas en un periodo dado, dividido entre el número de personas en el periodo (año). El denominador de las tasas se proyectó linealmente ${ }^{19}$ a partir de la población registrada en los censos generales de población de $1980,{ }^{20} 1990^{21}$ y $2000,{ }^{22}$ y en los conteos generales de población $1995^{23}$ y $2005 .^{24} \mathrm{La}$ temperatura máxima diaria se promedió por mes y por temporadas para cada uno de los años como sigue: temporada cálida de mayo a octubre y temporada fría de noviembre a abril. Se catalogaron como meses cálidos aquellos en los que la temperatura promedio fue superior a $30^{\circ} \mathrm{C} .^{11}$

Para evaluar los cambios de la tasa de suicidio en el tiempo, se utilizó el estudio de tendencia. Las tasas de suicidios se agruparon por temporadas (cálida o fría), así como por sexo. Previo a los análisis estadísticos, se evaluaron los supuestos de normalidad. Se utilizaron los análisis de varianza $t$ de Student y ANOVA para evaluar diferencias en la tasa de suicidio entre edades, temporadas y año, y la prueba de Tukey como prueba a posteriori para realizar las comparaciones múltiples. Para evaluar la tendencia de incremento en la tasa de suicidios, la temperatura y la relación entre ambas variables, se utilizaron modelos de regresión lineal y modelos cuadráticos de regresión con un valor de significancia de 
$\mathrm{p} \leq 0.05 .{ }^{5}$ El modelo cuadrático asume la posibilidad de que las tasas de suicidio cambien a lo largo de los años y que las tendencias de dicha variable se proyectan en una parábola en lugar de una tendencia lineal. Este modelo puede explicar los cambios por los cuales un incremento puede ser seguido de un decremento en las tasas de suicidio. ${ }^{5}$

Con el objetivo de evaluar el efecto de las variables ambientales (temperatura y temporada) sobre la tasa de suicidios registrada en BCS, y a su vez predecir la incidencia de suicidios en términos de dichas variables, se generó un modelo lineal generalizado (GLM, por sus siglas en inglés) con un error de Poisson y una función enlace logarítmica. ${ }^{25}$ La función de enlace log asegura que todos los valores que pueda tomar la variable dependiente (tasa de suicidios) sean positivos mientras que el error tipo Poisson toma en cuenta el hecho de que los datos son números enteros y que las varianzas son iguales a sus medias. ${ }^{26} \mathrm{Un}$ modelo generalizado mixto, un caso particular de los GLM, fue creado para evaluar la contribución del efecto aleatorio de la variable categórica "año" sobre las predicciones. ${ }^{26}$

Se generaron todos los modelos plausibles iniciando a partir de un modelo máximo, el cual incluyó todas las variables e interacciones de interés (temperatura*temporada). El término cuadrático de la variable "temperatura" también se incluyó en el análisis para evaluar su significancia en el ajuste del modelo. La selección del mejor modelo se realizó por medio de la comparación de la devianza residual, medida de discrepancia del ajuste del modelo, ${ }^{27}$ siguiendo el principio de parsimonia. ${ }^{26}$ Una vez seleccionado el modelo, se realizó un análisis de los residuales para investigar la capacidad de ajuste del mismo en relación con los datos observados. ${ }^{26}$ Los paquetes estadísticos utilizados fueron FOXPRO, Excel 2007, SPSS V13.0, Statistica v.7 y R v.2.14.0

\section{RESULTADOS}

Un total de 582 suicidios ocurrieron en BCS de 1985 a 2008. Las mujeres representaron el $9 \%(n=53$; promedio anual 2$)$ del total de suicidios, con una tasa promedio anual de 1.6 en cada 100000 mujeres (rango: 0 a 4.08). El 91\% de los casos $(\mathrm{n}=529$; promedio anual 22) correspondieron a suicidios realizados por hombres con una tasa promedio anual de 16.3 en cada 100000 hombres (rango: 4.58 a 24.17) (cuadro 1). Las mujeres fueron más jóvenes, con un promedio de 29.1 años (desviación estándar; $\mathrm{DE}=13.3$ ), con respecto a los hombres en los cuales la edad promedio al momento del evento fue de 37.3 años $(\mathrm{DE}=17.1)\left(\mathrm{F}_{(1,11)}=12.00 ; \mathrm{p}<0.01\right)$.

La tasa de suicidio fue significativamente mayor durante la temporada cálida en comparación con la temporada fría $\left(\mathrm{t}_{0.05,(2), 46}=-2.55, \mathrm{p}<0.01\right)$ (figura 1a). Durante los 24 años analizados en el presente estudio, se encontró una tendencia

Cuadro 1. Descripción de la mortalidad por suicidio en Baja California Sur y temperaturas máximas promedios según temporada cálida (mayo a octubre), temporada fría ((noviembre a abril) 1985 al 2008.

\begin{tabular}{|c|c|c|c|c|c|c|c|c|c|c|c|}
\hline \multirow[b]{2}{*}{ Año } & \multirow[b]{2}{*}{ Población* } & \multicolumn{4}{|c|}{ Suicidios (n) } & \multicolumn{4}{|c|}{ Tasa de suicidios** } & \multicolumn{2}{|c|}{$\mathrm{TMP}^{* * *}$} \\
\hline & & $\mathrm{H}$ & $M$ & $\mathrm{~F}$ & $\mathrm{C}$ & $\mathrm{H}$ & $M$ & $\mathrm{~F}$ & $\mathrm{C}$ & $\mathrm{F}$ & $\mathrm{C}$ \\
\hline 1985 & 195919 & 8 & 0 & 3 & 5 & 8.33 & 0.00 & 1.53 & 2.55 & 24.9 & 34.2 \\
\hline 1986 & 204901 & 7 & 1 & 3 & 5 & 6.97 & 0.96 & 1.46 & 2.44 & 26.2 & 33.7 \\
\hline 1987 & 213883 & 6 & 2 & 3 & 5 & 5.73 & 1.83 & 1.40 & 2.34 & 25.0 & 35.1 \\
\hline 1988 & 222864 & 5 & 0 & 3 & 2 & 4.58 & 0.00 & 1.35 & 0.90 & 26.1 & 34.3 \\
\hline 1989 & 231846 & 14 & 2 & 6 & 10 & 12.32 & 1.69 & 2.59 & 4.31 & 25.9 & 34.4 \\
\hline 1990 & 240828 & 16 & 0 & 7 & 9 & 13.56 & 0.00 & 2.91 & 3.74 & 24.7 & 33.5 \\
\hline 1991 & 242156 & 12 & 2 & 9 & 5 & 10.11 & 1.62 & 3.72 & 2.06 & 25.4 & 34.3 \\
\hline 1992 & 243483 & 15 & 1 & 7 & 9 & 12.57 & 0.81 & 2.87 & 3.70 & 26.0 & 36.3 \\
\hline 1993 & 244811 & 17 & 2 & 9 & 10 & 14.17 & 1.60 & 3.68 & 4.08 & 27.4 & 35.1 \\
\hline 1994 & 246138 & 11 & 1 & 1 & 11 & 9.12 & 0.80 & 0.41 & 4.47 & 27.3 & 36.7 \\
\hline 1995 & 247466 & 22 & 3 & 12 & 13 & 18.14 & 2.38 & 4.85 & 5.25 & 27.7 & 35.9 \\
\hline 1996 & 254970 & 28 & 1 & 16 & 13 & 22.41 & 0.77 & 6.28 & 5.10 & 28.6 & 35.9 \\
\hline 1997 & 262473 & 30 & 2 & 9 & 23 & 23.33 & 1.49 & 3.43 & 8.76 & 28.2 & 36.8 \\
\hline 1998 & 269977 & 30 & 3 & 16 & 17 & 22.68 & 2.18 & 5.93 & 6.30 & 28.1 & 36.8 \\
\hline 1999 & 277480 & 16 & 0 & 4 & 12 & 11.77 & 0.00 & 1.44 & 4.32 & 28.7 & 36.6 \\
\hline 2000 & 284984 & 20 & 1 & 6 & 15 & 14.32 & 0.69 & 2.11 & 5.26 & 27.9 & 35.9 \\
\hline 2001 & 296307 & 21 & 2 & 6 & 17 & 14.46 & 1.32 & 2.02 & 5.74 & 26.7 & 35.9 \\
\hline 2002 & 307629 & 34 & 4 & 17 & 21 & 22.56 & 2.55 & 5.53 & 6.83 & 28.1 & 37.1 \\
\hline 2003 & 318952 & 31 & 3 & 15 & 19 & 19.84 & 1.84 & 4.70 & 5.96 & 28.3 & 36.2 \\
\hline 2004 & 330274 & 30 & 4 & 11 & 23 & 18.54 & 2.37 & 3.33 & 6.96 & 27.4 & 36.9 \\
\hline 2005 & 341597 & 36 & 3 & 19 & 20 & 21.51 & 1.72 & 5.56 & 5.85 & 28.3 & 36.6 \\
\hline 2006 & 363109 & 43 & 4 & 17 & 30 & 24.17 & 2.16 & 4.68 & 8.26 & 28.9 & 37.0 \\
\hline 2007 & 384622 & 42 & 8 & 25 & 25 & 22.29 & 4.08 & 6.50 & 6.50 & 27.7 & 36.6 \\
\hline 2008 & 406134 & 35 & 4 & 20 & 19 & 17.59 & 1.93 & 4.92 & 4.68 & 29.1 & 36.1 \\
\hline
\end{tabular}

$\mathrm{H}=$ Hombres; $M=$ Mujeres; TMP = Temperatura máxima promedio.

* Población mayor de 14 años; ** Tasa de suicidios expresada en cada 100,000 habitantes; ** Temperatura expresada en grados centígrados. 

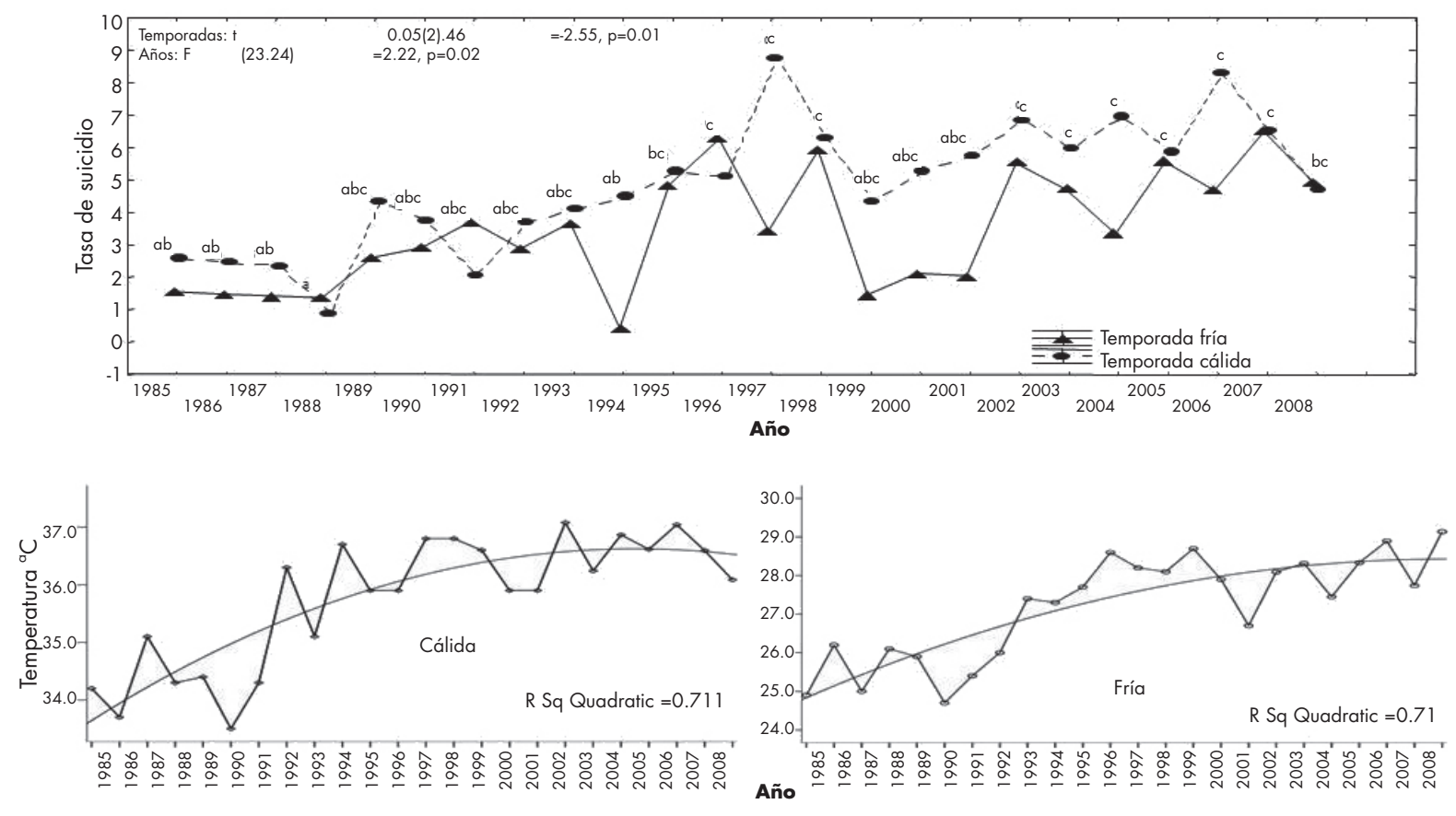

Figura 1. a) Tasa de suicidio por temporada cálida (mayo-octubre) y fría (noviembre a abril) por año en Baja California Sur. Las letras distintas sobre los puntos denotan diferencias significativas entre los años. b) Tendencias de las temperaturas por temporadas cálida (mayo a octubre) y fría (noviembre a abril) en Baja California Sur 1985 a 2008.

ascendente en la tasa de suicidio, tanto en los meses fríos como en los meses cálidos $\left(\mathrm{F}_{(23,24)}=2.22, \mathrm{p}=0.02\right)$ (figura 1a). La incidencia de suicidios presentó variaciones anuales, y se observó un incremento significativo a partir de 1996. La tasa de suicidios registrada de 1985 a 1988 fue significativamente menor en comparación con el periodo de 1996 a 2007 (Tukey, p<0.05) (figura 1a).

La tendencia de incremento en el número de suicidios a lo largo de los años durante la temporada cálida se explica con un modelo lineal $\left(\mathrm{R}^{2}=0.58, \mathrm{p}<0.01 ; \mathrm{F}_{(1,22)}=33.2\right)$. Sin embargo, el ajuste de los datos por medio de una regresión cuadrática $\left(R^{2}=0.64, p<0.01\right)$ fue el modelo que mejor explicó el fenóme- no en dicha temporada. En la temporada fría la tendencia de incremento también fue estadísticamente significativa y se observó por medio de una regresión lineal simple $\left(\mathrm{R}^{2}=0.38\right.$ $\left.\mathrm{p}<0.01 \mathrm{~F}_{(1,22)}=13.3\right)$. En esta temporada el modelo cuadrático no contribuyó en el ajuste de los datos de manera significativa en comparación al modelo lineal $\left(\mathrm{R}^{2}=0.38, \mathrm{p}<0.01\right)$.

Se analizó la tendencia de incremento de la tasa de suicidio a lo largo de los años considerando el sexo (figura 2). En el caso de las mujeres, el modelo cuadrático $\left(R^{2}=0.35 p<0.01\right)$ explicó mejor el fenómeno en un $6 \%$ en comparación con el modelo lineal $\left(\mathrm{R}^{2}=0.33, \mathrm{p}<0.01 \mathrm{~F}_{(1,22)}=6.7\right)$. En los hombres, el modelo cuadrático $\left(R^{2}=0.64, p<0.01\right)$ mejoró el ajuste en un

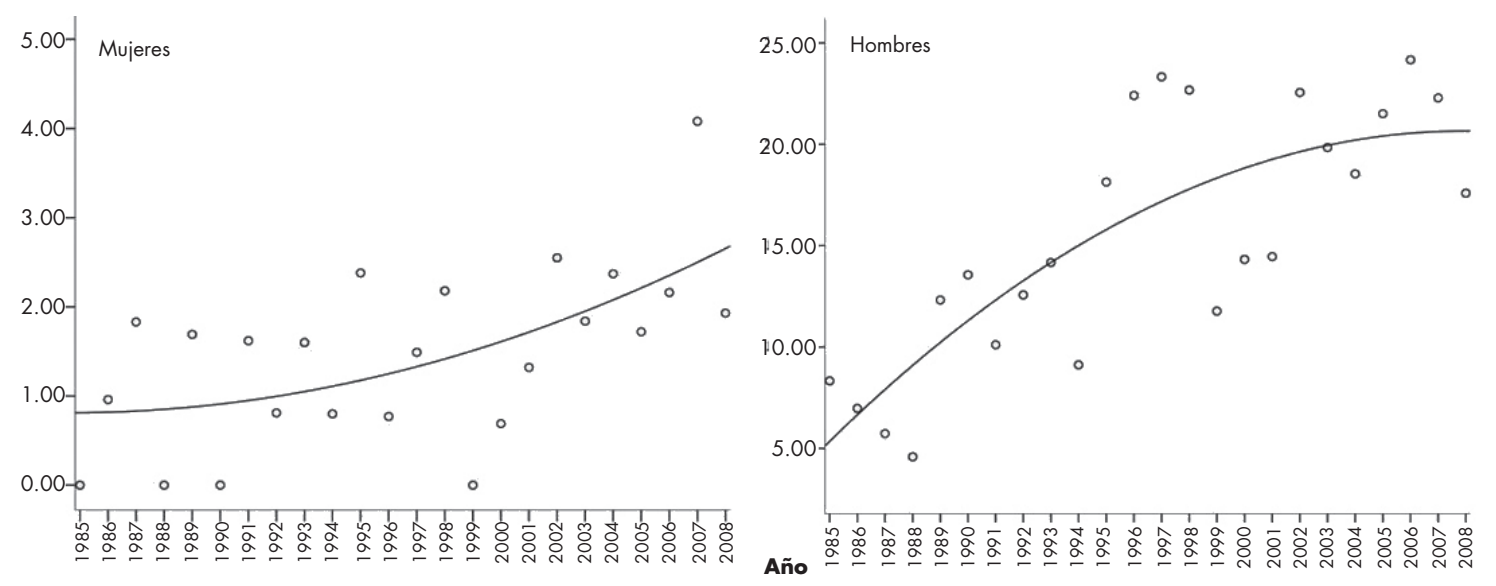

Figura 2. Tendencias de las tasas de suicidios por sexo en Baja California Sur 1985 a 2008. En el eje de las Y las tasas de suicidio por cada 100,000 habitantes; en el eje de las X los años. 
$8 \%$ en comparación al modelo lineal simple $\left(\mathrm{R}^{2}=0.59 \mathrm{p}<0.01\right.$, $\left.\mathrm{F}_{(1,21)}=33.2\right)$.

Al analizar el dato climático, se encontró un ascenso de la temperatura en ambas temporadas a lo largo de los años, con un incremento del $71 \%$ para ambas temporadas (modelo cuadrático $R^{2}=0.71 \mathrm{p}<0.01$ ) (figura $1 b$ ). Se halló una correlación positiva, estadísticamente significativa, entre la temperatura y las tasas de suicidio en ambas temporadas (cálida: $\mathrm{R}^{2}=0.68 \mathrm{p}<0.01, \mathrm{~F}_{(2,21)}=22.3$; fría: $\left.\mathrm{R}^{2}=0.033 \mathrm{p}<0.01, \mathrm{~F}_{(2,21)}=5.1\right)$.

El efecto aleatorio del año y el término cuadrático no fueron factores que contribuyeran significativamente en las predicciones de la tasa de suicido en los GLM. El modelo mínimo ajustado fue aquel que incluyó las variables temperatura ambiental y la estacionalidad, variables significativamente relacionadas con la tasa de suicidios. A partir de dichos resultados se generaron los modelos predictivos de la tasa de suicidios explicada por la variable temperatura para cada una de las temporadas:

$$
\begin{aligned}
& \text { T. suicidios frío }=\mathrm{e}^{-6.40+0.28 \text { Temperatura }} \\
& \text { T. suicidios cálido }=\mathrm{e}^{-8.42+0.28 \text { Temperatura }}
\end{aligned}
$$

Donde el modelo 1 predice la tasa de suicidios durante los meses de la temporada fría (noviembre a abril) y el modelo 2 genera predicciones durante la temporada cálida (mayo a octubre) en BCS (figura 3). Durante el análisis de los residuales se identificó que los modelos propuestos no predicen adecuadamente la tasa de suicidios cuando ésta presenta valores cercanos a cero (figura 3).

\section{DISCUSIÓN}

Este estudio exploratorio sugiere una relación entre el incremento de la temperatura ambiental y el número de suicidios registrados durante 24 años en BCS, México. Esta relación se encuentra bien definida en los meses cálidos, pero con una tendencia en aumento para los meses fríos, resultado de inviernos menos fríos y con una elevación de la temperatura durante este periodo. Este cambio climático ya ha sido reportado previamente por Gutiérrez-Ruacho. ${ }^{11}$ A nivel internacional existe evidencia del cambio climático y su relación con el evento suicida. En Europa los inviernos se han tornado menos fríos con un incremento de los suicidios durante esta época. ${ }^{5,28}$ Asimismo, el evento suicida es mayor en los hombres. Parte de este fenómeno pudiera ser atribuible a una posible acción de reducción de riesgo conferida por las hormonas esteroideas en las mujeres. En el caso de los hombres, es posible que los andrógenos participen en la conducta impulsiva que caracteriza el acto suicida o bien la falta de la posible acción protectora de la progesterona y el estradiol. ${ }^{29}$ Muchas otras explicaciones se han propuesto, ya que el patrón en cuanto al suicidio en el hombre durante los meses cálidos ha sido ampliamente reportado. ${ }^{6,30-33}$ Dentro de estas posibles explicaciones, la más plausible para BCS es la acción del calor sobre las vías que integran la percepción ambiental, información que es controlada en los circuitos de la memoria emocional, para de ahí pasar a la toma de decisiones. Las alteraciones funcionales de este circuito, aunadas a factores ambientales adversos, como el calor, parecen promover el acto suicida. Una de las principales entidades clínicas relacionadas con el suicidio es la depresión, pero no es la única, pues también son factores de riesgo el trastorno bipolar y el trastorno ezquizoafectivo. Cualquiera de los anteriores, aunados a la agitación e impulsividad causada por el estrés térmico, pueden estar detonando el evento suicida. 29,34

Preti (2007) relacionó el evento suicida en Italia con el calentamiento global. ${ }^{5}$ Comenta que existe una incapacidad en algunas porciones de la sociedad para responder a los cambios climáticos, en especial en los hombres, y que además puede incrementarse con la edad. En un trabajo previo

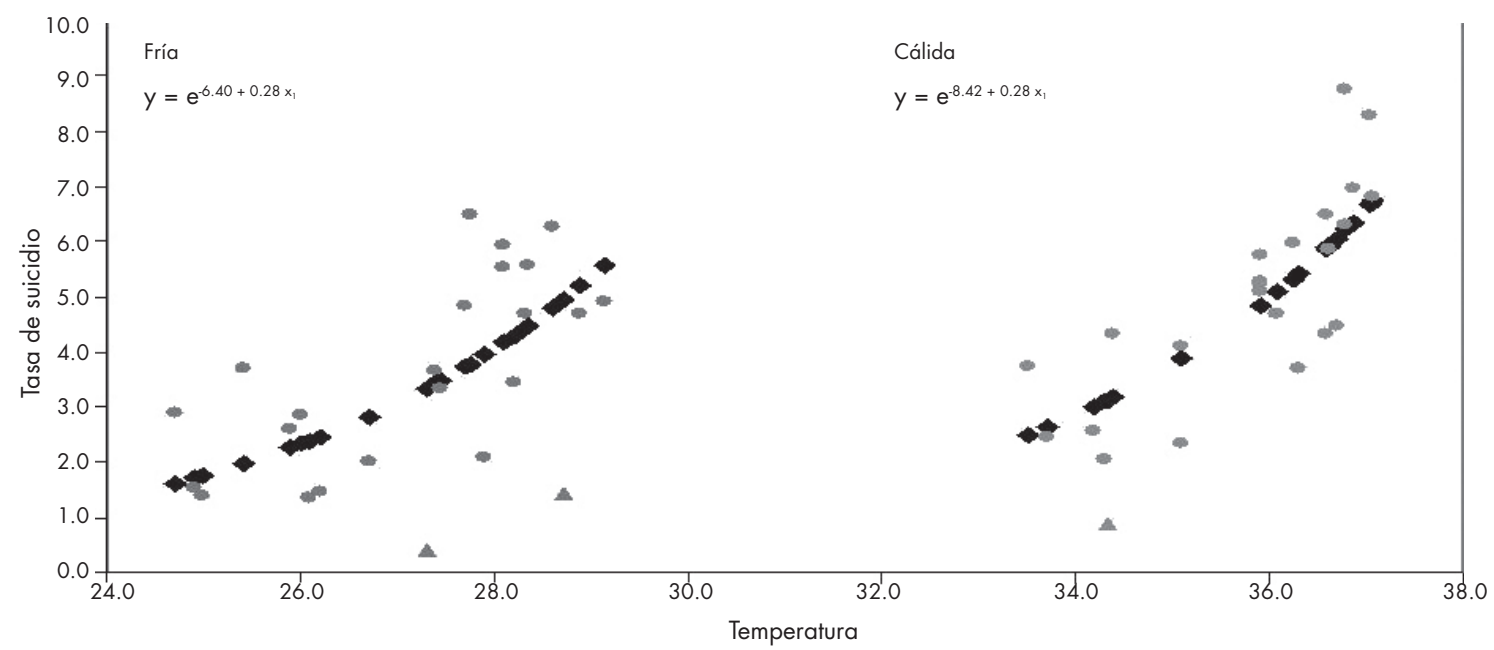

Figura 3. Tasa de suicidio en relación con la temperatura por temporada cálida (mayo-octubre) y fría (noviembre a abril)

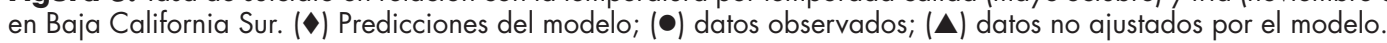


de 2009 con respecto al suicidio en la población de BCS se reporta un aumento del riesgo del suicidio en los hombres, por lo que lo comentado por Preti pudiera explicar la incapacidad para reaccionar ante el estrés y la falta de confort que el calor ocasiona. ${ }^{5,9}$ Esto se observa no sólo durante el verano sino también durante la época del frío que se torna cálida y se expresa en un incremento del evento suicida en esta porción de la sociedad en BCS. La tendencia del suicidio en los hombres de 1985 a 1997 fue ascendente, y de 1998 a 2008 se aprecia una reducción en los últimos 11 años. En contraposición, en los últimos 11 años se observa un incremento de la tendencia en los suicidios en las mujeres. Reportes similares se han presentado en Eslovenia y en Italia. ${ }^{35,36}$ Sus comentarios van dirigidos hacia un aumento de los suicidios en mujeres durante los meses de enero a junio en comparación con el periodo de julio a diciembre. Este patrón estacional obedece a episodios caracterizados por alteraciones del estado de ánimo más que por trastornos mentales. ${ }^{36}$

No se puede establecer una asociación unidireccional entre el evento suicida y el calor; múltiples factores intervienen en este fenómeno. En la sociedad de BCS, la falta de cohesión social y la protección que esto otorga es uno de los factores que tornan al individuo, sobre todo al hombre, más propenso al suicidio. ${ }^{9}$ Otra situación es el incremento de problemas de salud, por ejemplo, diferentes tipos de cáncer y enfermedades crónicas que pudieran precipitar también el evento suicida. ${ }^{37}$

En el presente trabajo se replicó el análisis estadístico utilizado por el equipo de Preti y se encontró consistencia en el método. La contribución original del presente estudio es el uso de los GLMs como herramienta estadística. Estos modelos son una buena opción, ya que consiguen eliminar los efectos de colinearidad en series altamente correlacionadas y con ellas estimar en una misma ecuación el efecto de la exposición. ${ }^{38}$ Con ello se integró un modelo predictivo de las tasas de suicidio con respecto a la temperatura ambiental para cada temporada. Las limitaciones en cuanto al método son propias de los estudios ecológicos, los cuales establecen la correlación a nivel grupal y no a nivel individual y el número reducido de variables que se tienen para su análisis. ${ }^{12}$ Otra situación que limita el presente estudio es establecer una relación entre las temperaturas promediadas mensuales, y posteriormente por temporada, y el evento suicida, el cual también se aglomeró en forma mensual y por temporada. Debido al limitado número de observaciones no es posible hacerlo de otra manera. A pesar de todos estos sesgos de información, se puede establecer una posible relación entre el incremento de la temperatura ambiental y el cambio climático que se ha experimentado en las últimas décadas y el suicidio.

\section{CONCLUSIÓN}

Es evidente el cambio climático que está experimentando la península de BCS y los potenciales efectos a la salud de sus pobladores. El presente trabajo demuestra la posible relación entre los suicidios y el incremento de la temperatura ambiental. Los dos modelos predictivos generados mostraron cómo la temperatura ambiental permite identificar la tendencia de la tasa de suicidios para ambas temporadas. Con este tipo de análisis se puede comparar si existe el mismo patrón con respecto al suicidio y la temperatura ambiental en otras regiones de México con alto riesgo de suicidio, que comparten con BCS el factor común de temperaturas ambientales altas.

\section{REFERENCIAS}

1. Sulman FG. The impact of weather on human health. Rev Environ Health 1984;4:83-119.

2. World Health Organization. Figures and facts about suicide. Technical report; Ginebra; 1999.

3. Levi F, Vecchina C, Luchini F, Negri E et al. Trends in mortality from suicide, 1965-1999. Acta Psychiatr Scand 2003;108:341-349.

4. McMichael AJ, Woodruff RE, Hals S. Climate change and human health: present and future risks. Lancet 2006;368: 859-869.

5. Preti A, Lentini G, Maugeri M. Global warming possibly linked to an enhanced risk of suicide: Data from Italy, 1974-2003. J Affect Disord 2007;102:19-25.

6. Page LA, Hajat S, Kovts RS. Relationship between daily suicide counts and temperature in England and Wales. Br J Psychiatry 2007;191:106-112.

7. Salib E, Cortina-Borja M \& Anderson D. Hot weather and suicide: a real risk o statistical illusion? Br J Psychiatry 2007;191:560-561.

8. Hijar M, Rascon RA, Blanco J, López VM. Los suicidios en México. Características sexuales y geográficas (1973-1993). Salud Mental 1996;19:14-21.

9. Gaxiola-Robles R, Bitzer-Quintero OK, García-González A, Celis A. El estado civil y el suicidio en Baja California Sur. Rev Med Inst Mex Seguro Soc 2009;47:383-386.

10. Instituto Nacional de Estadística, Geografía e Informática. Marco geoestadístico municipal; México; 2005.

11. Gutiérrez-Ruacho OG, Brito-Castillo L, Díaz-Castro SC, Watts CJ. Trends in rainfall and extreme temperature in northwestern Mexico. Clim Res 2010;42:133-142.

12. Laurier D, Valenty $M$, Tirmarche $M$. Radon exposure and the risk of leukemia: a review of epidemiological studies. Health Phys 2001;81: 272-288.

13. Sistema Nacional de Información en Salud. Mortalidad. México. Secretaría de Salud; 2010. Hallado:http://sinais.salud.gob.mx/basesdedatos/defunciones.html

14. Organización Mundial de la Salud. Clasificación estadística internacional de enfermedades, traumatismos y causas de defunción. Novena revisión. Washington, DC: Organización Panamericana de la Salud; 1978.

15. Organización Mundial de la Salud. Clasificación estadística internacional de enfermedades y problemas relacionados con la salud. Décima revisión. Washington, DC: Organización Panamericana de la Salud; 1998.

16. Maris RW. Suicide. Lancet 2002;360:319-326.

17. Mann J. A current perspective of suicide and attempted suicide. Ann Intern Med 2002;136:302-311.

18. Instituto Mexicano de Tecnología del Agua. Extracto Rápido de Información Climatológica ERIC III. CD ROM. México; 2008.

19. Camel F. Estadística médica y planificación en salud. Vol. II. Mérida, Venezuela: Consejo de Publicaciones de la Universidad de los Andes; 1982.

20. Instituto Nacional de Estadística, Geografía e Informática. Censo general de población y vivienda, 1980. México; 1984.

21. Instituto Nacional de Estadística, Geografía e Informática. Resumen general. XI Censo general de población y vivienda. Aguascalientes; 1992. 
22. Instituto Nacional de Estadística, Geografía e Informática. XII Censo general de población y vivienda, 2000 [base de datos en CD]. Aguascalientes, México; 2001.

23. Instituto Nacional de Estadística, Geografía e Informática. Conteo de población y vivienda 1995. Resultados definitivos. Tabuladores básicos. Aguascalientes; 2007.

24. Instituto Nacional de Estadística, Geografía e Informática. II Conteo de población y vivienda 2005. Resultados definitivos. Hallado: http://www. inegi.org.mx/est/contenidos/proyectos/ccpv/cpv2005/Default.aspx

25. Puig X, Ginebra J, Gispert R. Análisis de la evolución temporal de la mortalidad mediante modelos lineales generalizados. Gac Sanit 2005;19:481-485.

26. Crawley MJ. The R Book. England: John Wiley and Sons Ltd; 2007.

27. McCullagh $P$, Nelder JA. Generalized linear models. Florida: Chapman and Hall/CRC; 1989.

28. Brunetti M, Maugeri M, Monti F, Nanni T. Temperature and precipitation variability in Italy in the last two centuries from homogenized instrumental time series. Int J Climatol 2006;26:345-381.

29. Gutiérrez-García AG, Contreras CM. El suicidio y algunos de sus correlatos neurobiológicos. Primera parte. Salud Mental 2008;31:321-330.

30. Salib E, Gray N. Weather conditions and fatal self harm in North Cheshire 1989-1993. Br J Psychiatry 1997;171:473-477.
31. Hakko $H$, Räsänen $P$, Tiihonen J. Seasonal variation in suicide occurrence in Finland. Acta Psychiatr Scand 1998;98:92-97.

32. Preti A, Miotto P. Seasonality in suicides: the influence of suicide method, gender and age on suicide distribution in Italy. Psychiatry Res 1998;81:219-231.

33. Preti A. The influence of climate on suicidal behavior in Italy. Psychiatry Res 1998;78:9-19.

34. Spreux-Varoquaux O, Alvarez JC, Berlin I, Batista G et al. Differential abnormalities in plasma 5-HIAA and platelet serotonin concentrations in violent suicide attempters relationships with impulsivity and depression. Life Sci 2001;69:647-657.

35. Oravecz R, Sisti D, Rocchi MB, Preti A. Changes in the seasonality of suicide over time in Slovenia, 197 to 2002 . Amplitude is only positively related to suicide rates among females. J Affect Disord 2007;104:211-215.

36. Rocchi MB, Sisti D, Miotto P, Preti A. Seasonality of suicide: relationship with the reason of suicide. Neuropsychobiology 2007;56:86-92.

37. Misono S, Weiss NS, Fann JR, Redman M et al. Incidence of suicide in persons with cancer. J Clin Oncol 2008;26:4731-4738.

38. Muñoz F, Carvalho MS. Effect of exposure time to PM(10) on emergency admissions for acute bronchitis. Cad Saude Publica 2009;25:529-539.

Artículo sin conflicto de intereses 